\title{
Reducing global mortality from childhood diarrhea: future directions
}

\author{
“There are two broad ways to reduce the burden of childhood diarrheal \\ diseases: first, through reducing the incidence by preventing transmission of \\ pathogens and establishing resistance to infections by specific pathogens; \\ and second, through improved case management."
}

Diarrheal disease is currently the second leading contributor to the global 10.4 million deaths of children under 5 years of age each year. Young children suffer from a median of 3.2 episodes with an estimated 2 billion global episodes leading to nearly 2 million deaths, and developing countries share over $80 \%$ of these deaths [1,2]. Persistent diarrhea contributes $45 \%$ of these deaths, followed by acute watery diarrhea $(35 \%)$ and dysentery (20\%) [3]. Frequent diarrhea episodes in young age are also a major contributor to malnutrition, with the impaired host defense making children vulnerable to further attacks of infectious diseases, thus impeding their development. The high disease burden of childhood diarrhea is associated with significant healthcare costs, both at provider and societal levels.

“A situation analysis revealed that 6 million child deaths could be prevented if 23 already proven interventions could be applied in 42 countries that together contribute $90 \%$ of the child deaths."

The fourth Millennium Development Goal (MDG) aims to reduce global deaths of children under 5 years of age by two-thirds between the years 1990 and 2015. However, child deaths have reduced from 13 million in 1980 to a current level of 10 million; at this rate of decline only $27 \%$ out of the targeted $67 \%$ reduction in child deaths would be possible by the year 2015 [4], suggesting that many developing countries will not achieve the goal unless special efforts are made. There are many contributors to child deaths, and thus prioritization is necessary in achieving the goal - a smaller proportionate reduction from greater contributors would pay back in a greater way.
Do we need research to reduce the diarrhea disease burden, particularly to achieve the MDG 4, and if we do, what types of research will be needed? Research investments, including those for diarrheal diseases, have paid back mostly in terms of knowledge production and finding effective interventions, and there is a relative lack of translational research for successful implementation of research findings with wider coverage. A situation analysis revealed that 6 million child deaths could be prevented if 23 already proven interventions could be applied in 42 countries that together contribute $90 \%$ of the child deaths [5], while another review concluded that even the resource-poor countries could reduce child deaths by two-thirds - which is the current target for MDG 4 - by improving coverage of available interventions [6].

The risk factors for diarrhea and its severity and deaths, such as young age, malnutrition, nonexclusive breastfeeding, poor hand washing and hygienic practices, lack of measles immunization and zinc deficiency, are similar to that of pneumonia [7]. Therefore, addressing them effectively would have a great impact on child deaths.

The etiology of diarrhea is diverse; however, nearly all childhood episodes in developing countries (except those due to inborn errors of metabolism) are caused by infectious agents, suggesting that solutions could be found within the paradigm of infectious diseases. Rotavirus, enterotoxigenic Escherichia coli (ETEC), Shigella, Salmonella and Vibrio cholerae are the most important diarrheal pathogens, with rotavirus the leading cause both in developing and developed countries, accounting for over half a million deaths [8].

There are two broad ways to reduce the burden of childhood diarrheal diseases: first, through reducing the incidence by preventing transmission of pathogens and establishing resistance to infections by specific pathogens; and second, through improved case management.
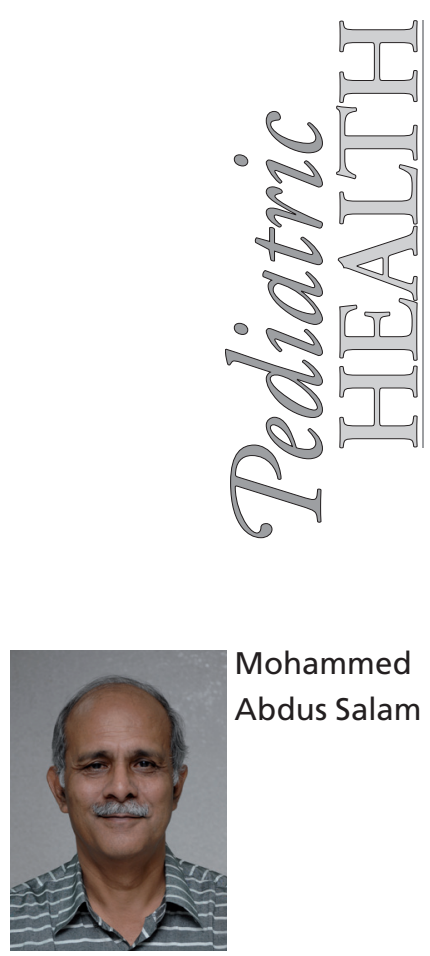

Mohammed Abdus Salam

Director, Clinical Sciences Division International Centre for Diarrhoeal Disease Research, Bangladesh (ICDDR, B), 68 Shaheed Tajuddin Ahmed Sarani, Mohakhali, Dhaka 1212, Bangladesh

Tel.: +88029882 399

Fax: +880298856757

masalam@icddrb.org

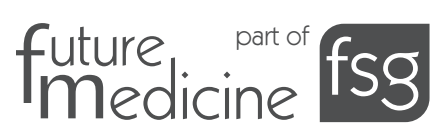


Prevention of transmission of diarrheal pathogens requires special emphasis owing to its potential to reduce incidence and thereby deaths. The preventive measures can additionally impact other enteric and nonenteric infectious diseases, such as typhoid fever, hepatitis and pneumonia. Diarrheal pathogens are transmitted through the fecal-oral route, and contaminated water and food are the most important vehicles. Infants, undernourished and nonbreastfed children are at greater risk of food borne and other infectious diseases, including diarrheal diseases and pneumonia, and they are also at higher risk of severe and prolonged illness, as well as at higher risk of death [9]. Undernutrition and suboptimal breastfeeding have been identified as the underlying causes in 30 and $5 \%$ of all deaths, respectively [10].

\section{"Effective and robust interventions are available for both case management, including oral rehydration solution and zinc therapy, and also for prevention, including control of the \\ environment, improved sanitation, exclusive breastfeeding, hand washing and vaccines, to help achieve the MDG 4."}

Among preventive strategies, exclusive breastfeeding during the first 6 months of life, and its continuation along with appropriate and safe complementary food, is important. Breast milk is an ideal food that meets the nutrient requirements of young infants, provides antibodies to protect them against infectious diseases and reduces their exposure to contaminated food and water prevailing in most developing countries; continuation of breastfeeding for 13 months or longer significantly reduces incidence of diarrheal diseases [11]. The risk of overall and diarrheal deaths is 2.5 - and 3.5-times greater, respectively, in partially breastfed, and 10.5- and 9-times greater, respectively, among nonbreastfed children [12]. It is estimated that breastfeeding can prevent $13 \%$ of deaths and appropriate complementary feeding can prevent another $6 \%$ of deaths (i.e., together nearly onefifth of total child deaths) [13], including those due to pneumonia and neonatal sepsis [6]. A review of survey data from ten countries in South-East Asia and Asia-Pacific observed that the rate of exclusive breastfeeding during 2-3 months of age varied from 7.6 to $56 \%$, which sharply declines to $0-30.6 \%$ by $4-5$ months of age - calling for an improvement in the situation [101].
Most developing countries feature contaminated environments that facilitate infectious diseases, where people, including infants and young children, are exposed to water that is potentially contaminated with enteric pathogens, including those causing diarrheal diseases. Lack of sanitation and hygienic practices are important contributors to diarrheal diseases, and they have lowest coverage in developing countries, particularly in Asia and Africa, and over 1 billion people currently do not have access to clean water. It has been estimated that interventions in water supply, sanitation and hygiene can reduce diarrhea incidence by a quarter $(25 \%)$ and child mortality by $65 \%$ [102]. Appropriate hand washing with soap is a robust intervention that has been observed to reduce the incidence of both diarrhea and pneumonia by at least $50 \%$, and is effective when practiced in contaminated environments $[13,14,103]$.

A vaccine approach is a cost-effective intervention in controlling infectious diseases; however, development of effective vaccines against important diarrheal pathogens has been much slower, and only recently have oral vaccines become available for the prevention of rotavirus infection and/or to reduce the severity of diarrhea and cholera. Rotavirus vaccines have been proven effective and safe in industrialized countries and Latin America; however, owing to the need for further assessment of their effectiveness and safety in other developing countries, the WHO does not recommend their routine global use [8]. An oral vaccine is also available for prevention of cholera; however, its higher cost is prohibitive for its routine application in most resource-constrained developing countries, while evaluation of other cheaper vaccines is underway.

Improved case management is another strategy for reducing deaths. Therapies for diarrheal diseases, irrespective of etiology, include management (prevention and treatment) of dehydration, continued feeding (including breastfeeding) and effective antimicrobial therapy for selective indications, such as shigellosis, severe cholera, and invasive salmonellosis and intestinal amoebiasis. No pharmacological therapy is currently available for the treatment of rotavirus diarrhea - the leading cause of diarrhea globally. It has been estimated that optimal use of oral rehydration solution (ORS) can reduce the current number of deaths of children under 5 years of age by an impressive $15 \%$, zinc treatment by $5 \%$ and antimicrobial therapy for dysentery by $3 \%$ [6]. Put together, these three interventions could make a substantial reduction in child deaths. 
The great reduction in annual diarrheal deaths from approximately 4.5 million in 1980 to the current less than 2 million has largely been possible owing to the promotion of use of ORS [15]. Dehydration accounts for $60 \%$ of diarrheal deaths, and the impressive reduction has been ascribed to oral rehydration therapy (ORT) using recommended home fluids and ORS. Its potential to reduce deaths further comes from the observations that the global use of ORS is still suboptimal - data from 68 UNICEF priority countries indicate a median use of ORS for $38 \%$ of the episodes [16], representing only a $2 \%$ increase between 2000 and 2006 [17]. One of the frequently cited reasons for lower use of ORT, despite its universal awareness, is its failure to reduce duration or severity of diarrhea.

"Control of the environment and improvements in water supply and sanitation would require resources that most developing countries lack, and despite their importance might be seen as long-term strategies."

Zinc, currently recommended for routine use in the case management of diarrheal diseases irrespective of etiology, has both therapeutic and preventive effects. Zinc therapy reduces both the duration of diarrhea and likelihood of a prolonged episode, as well as stool volume, by $25 \%$ or greater, and the risk of death from persistent diarrhea by over $40 \%$ - the greatest contributor of diarrheal deaths [18]. Zinc supplementation, on the other hand, reduces the incidence and prevalence of diarrhea by $11-18 \%$ and $25-34 \%$, respectively, and additionally reduces antibiotic use by $70 \%[18]$. It is possible to provide zinc at a low cost that is likely to be affordable by even developing countries.

Antimicrobial therapy is among the options in the management of infectious diseases. However, such therapy is not recommended for rotavirus - the most important global pathogen for childhood diarrhea. Nitazoxanide has been assessed as effective in the management of rotavirus diarrhea in some trials, but requires further evaluation for its general acceptance. ETEC is another important childhood diarrheal pathogen, but antimicrobial therapy is not indicated as they are usually resistant to commonly used agents. Antimicrobial therapy is of proven efficacy in the treatment of severe cholera and shigellosis, and also invasive salmonellosis, but such therapy is complicated by the emergence of multiply resistant organisms. In Bangladesh and many other developing countries, $V$. cholerae $\mathrm{O} 1$ is currently resistant to all known effective drugs except azithromycin. In most localities, Shigellae are resistant to ampicillin, trimethoprim-sulphamethoxazole and nalidixic acid and demonstrates higher minimum inhibitory concentration to frank resistance for newer quinolones such as ciprofloxacin. The prevalence of Shigella dysenteriae type 1 , the serotype that is associated with more severe diseases and more complications including bacteremia, hypoglycemia and hemolytic uremic syndrome, and acquires resistance rapidly, has been reported to be decreasing including its apparent elimination from India and Bangladesh [104]. However, antimicrobial therapy for shigellosis remains a problem.

Effective and robust interventions are available for both case management, including ORS and zinc therapy, and also for prevention, including control of the environment, improved sanitation, exclusive breastfeeding, hand washing and vaccines, to help achieve the MDG 4. Globally, 2.4 billion people, mostly in developing countries, do not have access to improved sanitation facilities, and little progress has been made in the last decade [105]. Control of the environment and improvements in water supply and sanitation would require resources that most developing countries lack, and despite their importance might be seen as long-term strategies.

“...case studies with ORS and zinc have clearly demonstrated that despite high levels of awareness about ORS and zinc, the use rate is dismally low."

Exclusive breastfeeding during the first 6 months and its continuation until 24 months of age along with appropriate complementary feeding and hand washing are the two key preventive interventions that will not only reduce the global disease burden due to diarrhea, but also of pneumonia, the two leading causes of child death. These efforts need to be complemented by development of vaccines that are affordable to developing countries. In addition, their incorporation within the existing Expanded Programme for Immunization, with support from The Global Alliance for Vaccines and Immunisation, is another robust intervention to reduce global disease burden. 
Improved case management, in particular routine use of ORS and zinc therapy, and effective antimicrobial therapy for specific indications will remain important to prevent deaths and reduce incidence of morbidity close to none; however, their coverage is suboptimal $[16,17]$. Routine zinc therapy was recommended in 2004. A national program in Bangladesh could create near universal (90\%) awareness within 2 years, yet it is used in less than $20 \%$ of the episodes. Similarly, despite recognition of the importance of breastfeeding and hand washing, their actual practice varies widely and remains far too low to impact the incidence of diarrheal diseases. These findings clearly highlight that awareness is not enough - measures are necessary for improving the rate of practice. Efforts are being continued to find an effective antisectretory agent, and finding one would help reduce morbidity and deaths from diarrhea caused by $V$. cholerae and ETEC.

\section{"Scaling up health delivery is the challenge, and the lack of funds will be the limiting factor in reducing child mortality by two-thirds by 2015."}

A recent article summarized health research priority issues identified by a group of experts; health system and policy research topped the list with the aim to identify the barriers to implementation of the available and effective interventions, specifically to improve acceptability and effectiveness of ORS and zinc as case-management strategies [19]. The costeffectiveness ratio (CER) of ORS in averting each disability-adjusted life year (DALY) and child death has been estimated at US\$113 and $\$ 3200$, respectively. In the management of all acute diarrhea, the CER of zinc for averting each DALY and child death has been estimated to be only US $\$ 11$ and $\$ 307$, respectively [1]. The national policy planners need to be informed and convinced about the cost-effectiveness of ORT and routine zinc therapy for the treatment of diarrhea on diarrheal morbidity and deaths. The case studies with ORS and zinc have clearly demonstrated that despite high levels of awareness about ORS and zinc, the use rate is dismally low; dealing only with supply side issues would not be adequate in achieving the full benefit of these interventions and, thus, it is important to deal with demand side issues (i.e., creating demand for effective interventions).

“Although per capita expenditure of only US $\$ 1.23$ is required to save the life of a child, most of the resource-poor countries will require external funding support to achieve the MDG 4."

Anthropological studies to understand the barriers and identifying effective strategies to overcome them will be critical for implementation of proven interventions, which would require adequate funding. An estimated $\$ 5$ billion per year would be required to save the lives of 6 million children in over 42 countries where $90 \%$ of child deaths occur. Scaling up health delivery is the challenge, and the lack of funds will be the limiting factor in reducing child mortality by two-thirds by 2015 [20]. Although per capita expenditure of only $\$ 1.23$ is required to save the life of a child, most of the resource-poor countries will require external funding support to achieve the MDG 4.

\section{Financial \& competing interests disclosure}

The author has no relevant affiliations or financial involvement with any organization or entity with a financial interest in or financial conflict with the subject matter or materials discussed in the manuscript. This includes employment, consultancies, honoraria, stock ownership or options, expert testimony, grants or patents received or pending, or royalties.

No writing assistance was utilized in the production of this manuscript.

\section{Bibliography}

1. Robberstad B, Strand T, Black RE, Sommerfelt H: Cost-effectiveness of zinc as adjunct therapy for diarrhoea in developing countries. Bull. World Health Organ. 82, 523-531 (2004).

2. Kosek M, Bern C, Guerrant RL: The global burden of diarrhoeal disease, as estimated from studies published between 1992 and
2000. Bull. World Health Organ. 81, 197-204 (2003).

3. Black RE: Persistent diarrhea in children in developing countries. Pediatr. Infect. Dis. J. 12, 751-762 (1993).

4. Murray CJ, Laakso T, Shibuya K, Hill K, Lopez AD: Can we achieve Millennium Development Goal 4? New analysis of country trends and forecasts of under- 5 mortality to 2015. Lancet 370, 1040-1054 (2007).
5. The Bellagio Study Group on Child Survival: Knowledge into action for child survival. Lancet 362, 323-327 (2003).

6. Jones G, Steketee RW, Black RE, Bhutta ZA, Morris SS; Bellagio Child Survival Study Group: How many child deaths can we prevent this year? Child survival II. Lancet 362, 65-71 (2003). 
7. Rudan I, Boschi-Pinto C, Biloglav Z, Mulholland K, Campbell H: Epidemiology and etiology of childhood pneumonia. Bull. World Health Organ. 86, 321-416 (2008).

8. Weekly epidemiological report. World Health Organization. 82, 285-296 (2007).

9. Food borne disease: a focus for health education. Who 2000; Children's health and environment: a review of evidence. WHO Regional office for Europe (2002).

10. Black RE, Allen LH, Bhutta $Z$ et al.: Maternal and child undernutrition: global and regional exposures and health consequences. Lancet 371, 243-260 (2008).

11. Howie PW, Forsyth JS, Ogston SA, Clark A, Florey C: Protective effect of breastfeeding against infections. BMJ 300, 11-16 (1990).

12. Bahl R, Frost C, Kirkwood BR et al.: Infant feeding patterns and risks of death and hospitalization in the first half of infancy: multicentre cohort study. Bull. World Health Organ. 83(6) 418-426 (2005).

13. Luby SP, Agboatwalla M, Painter J, Altaf A, Billhimer WL, Hoekstra RM: Effect of intensive handwashing promotion on childhood diarrhea in high-risk communities in Pakistan. A randomized controlled trial. JAMA 291, 2547-2554 (2005).
14. Luby S, Agboatwalla M, Raza A et al.: A low cost intervention for cleaner drinking water in Karachi, Pakistan. Int. J. Infect. Dis. 5, 144-150 (2001).

15. The magnitude of the global problem of acute diarrhoeal disease: a review of active surveillance data. Bull. World Health Organ. 60, 605-613 (1984).

16. Bryce J, Terreri N, Victora CG et al.: Countdown to 2015: tracking intervention coverage for child survival. Lancet 368, 1067-1076 (2006).

17. Bryce J, Daelmans B, Dwivedi A et al.; Countdown Coverage Writing Group; Countdown to 2015 Core Group: Countdown to 2015 for maternal, newborn, and child survival; the 2008 report on tracking coverage of interventions. Lancet 371, 1247-1258 (2008)

18. Guidelines for policy makers and programme managers. World Health Organization (2006).

19. Fontaine $\mathrm{O}$, Kosek M, Bhatnagar $S$ et al.: Setting research priorities to reduce global mortality from childhood diarrhoea by 2015 . PLoS Med. 6(3), E1000041 (2009).

20. Bryce J, Black RE, Walker N, Bhutta ZA, Lawn JE, Steketee RW: Can the world afford to save the lives of 6 million children each year? Lancet 365, 2193-2200 (2005).

\section{Websites}

101. The breastfeeding scene in the South-East Asia region. Nutrition for Health and Development.

www.searo.who.int/LinkFiles/Nutrition_for_ Health_and_Development_Breastfeeding_ scene_SEAR.pdf

102. Lack of water and inadequate sanitation. www.who.int/entity/ceh/risks/cehwater/en

103. WHO guidelines on hand hygiene in health care; executive summary. www.who.int/patientsafety/challenge/en

104. Diarrhoeal Diseases (Updated February 2009). who.int/vaccine_research/diseases/ diarrhoeal/en/index $5 . h t m l$

105. Lack of water and inadequate sanitation. Children's environmental health. www.who.int/topics/water/en

\section{Affiliation}

- Mohammed Abdus Salam Director, Clinical Sciences Division, International Centre for Diarrhoeal Disease Research, Bangladesh (ICDDR, B), 68 Shaheed Tajuddin Ahmed Sarani, Mohakhali, Dhaka 1212, Bangladesh

Tel.: +88 029882399

Fax: +880298856757 masalam@icddrb.org 